\title{
Slip transfer through a general high angle grain boundary in nanocrystalline aluminum
}

\author{
C. Brandl, E. Bitzek, P. M. Derlet, and H. Van Swygenhoven ${ }^{\mathrm{a})}$ \\ ASQ/NUM-Material Science and Simulation, Paul Scherrer Institute, PSI-Villigen CH-5232, Switzerland
}

(Received 1 June 2007; accepted 23 August 2007; published online 14 September 2007)

The atomistic details of a slip transfer through a general high angle grain boundary in three dimensional nanocrystalline $\mathrm{Al}$ are reported and discussed in terms of possible implications for mesoscopic simulation models. (C) 2007 American Institute of Physics. [DOI: 10.1063/1.2784939]

The interaction of lattice dislocations with grain boundaries (GBs) determines to a large extent the mechanical behavior of polycrystalline materials, especially when the grain size is reduced to the nanometer regime. ${ }^{1,2}$ Experimentally, several mechanisms for slip propagation by dislocation movement across grain boundaries have been observed: ${ }^{1,3}$ (a) direct transmission of slip, (b) direct transmission with creation of a residual Burgers vector, (c) indirect transmission (possibly combined with reflection of the incoming dislocation), and (d) deposition or absorption of the dislocation at the grain boundary. For direct transmission of dislocations, the involved slip systems in both grains have to intersect along a common line in the boundary. ${ }^{1}$ In this geometry, dislocation/grain boundary interactions have been addressed extensively by molecular dynamics performed on bicrystals. $^{4-9}$ On the other hand, large scale molecular dynamics are also used to study the deformation mechanism of three dimensional (3D) nanocrystalline samples. ${ }^{10,11}$ These simulations showed that the grain boundaries act as both source and sink of dislocations. However, the transmission of slip through general high angle grain boundaries has not been investigated until now.

In this letter we report on the observation of slip transmission through a general high angle grain boundary during tensile deformation of a fully 3D nanocrystalline sample, and discuss implications for the mesoscopic modeling of dislocation/grain boundary interactions.

Molecular dynamics (MD) simulations at $300 \mathrm{~K}$ and a constant tensile stress of $1.6 \mathrm{GPa}$ were performed on a nanocrystalline Al sample containing 15 randomly oriented grains with a mean grain size of $12 \mathrm{~nm}$. The sample was produced using the Voronoi construction following the procedure detailed in Ref. 12. The atomic interaction was modeled by the embedded atom model potential for $\mathrm{Al}$ of Mishin et al. ${ }^{13}$ The atomic configurations were analyzed using the medium range order (MRO) according to the Honeycutt and Andersen procedure ${ }^{14}$ The MRO color visualization used in Figs. 1-3 encodes an atom local symmetry where gray represents facecentered cubic (fcc), red hexagonal closed packed (hcp), green other 12-coordinated, and blue non-12-coordinated atoms. Burgers vectors were determined using a slip vector analysis procedure. ${ }^{15}$ Burgers vectors and glide planes are identified using the usual Thompson tetrahedron notation. ${ }^{16}$

Figure 1(a) shows the dislocation/grain boundary interaction at the boundary between the grains 9 and 11. The relative orientation of the slip systems of both grains is

${ }^{a)}$ Electronic mail: helena.vs@psi.ch shown in Fig. 1(b) together with the identified Burgers vectors on the Thompson tetrahedra. The atomic structure of the general high angle grain boundary is shown in Fig. 2, where Fig. 2(a) demonstrates the $22^{\circ}$ tilt angle and Fig. 2(b) the $8^{\circ}$ twist angle of the interface. The orientation relationship does not correspond to a special misorientation. One can, however, observe in both figures coherent regions (yellow) separated by regions of atomic misfit, which due to the finite twist angle [Fig. 2(b)], are not columnar but instead exist as a patchwork of finite regions within the GB plane. The coherent regions are located around the intersection points between the $(-1-11)_{9}[(c)$ plane in Thompson tetrahedron notation] plane of grain 9 and the $(1-11)_{11}(a)$ plane of grain 11. In these coherent regions the change of direction of the close packed [101] rows is continuous [see Fig. 2(a)], and the atoms within the close packed $(c)_{9}$ and $(a)_{11}$ planes locally assume a close to identical orientation [Fig. 2(b)]. A dislocation on a $(c)$-plane in grain 9 will thus encounter a number of coherent regions as it propagates through the grain. It is one such dislocation that is studied in the present work.

During tensile deformation at constant stress, a dislocation $A D(c)$ is nucleated close to a triple junction in grain 9 and travels through the grain on a slip system with a high Schmid factor $m=0.4$, depositing on its way dislocation segments of predominantly edge character at the GB 9/11. The details of the interaction between $A D(c)_{9}$ and the grain boundary to grain 11 are shown in a series of snapshots of the same region in Fig. 3. When the full dislocation passes the first coherent region, a partial dislocation $D \alpha(a)_{11}$ appears on the other side of the grain boundary in grain 11 [see Fig. 3(a)]. In the following snapshot [Fig. 3(b)], the partial dislocation segment in grain 11 disappears. During the passage of the next coherent GB region by the dislocation $A D(c)$
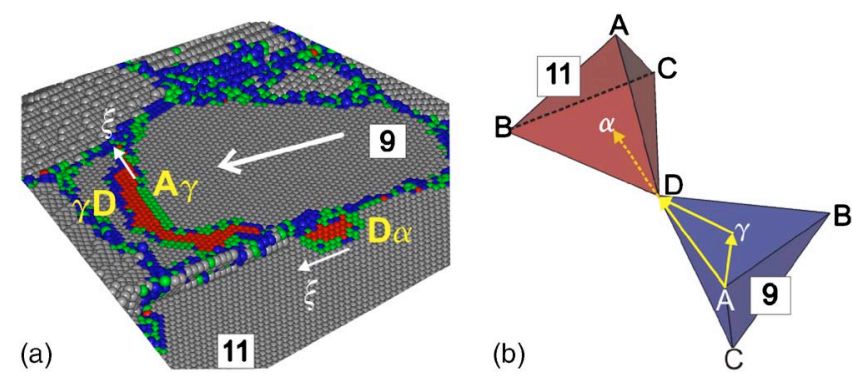

FIG. 1. (Color online) (a) Dislocation configuration after dislocation passage in grain 9 and slip transfer to grain 11. (b) Relative orientation of the slip systems of the two grains with the identified Burgers vectors of the dislocations. 

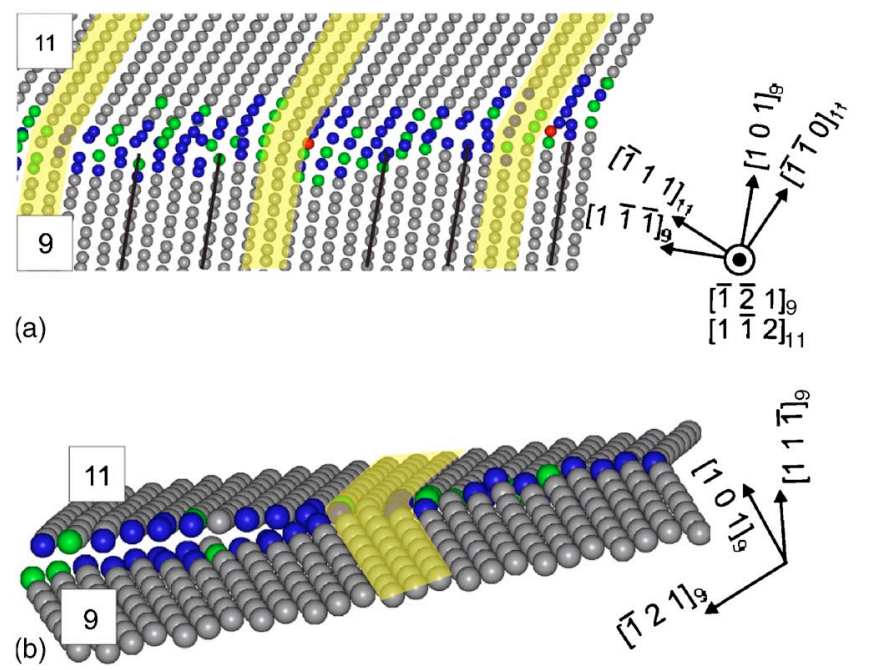

FIG. 2. (Color online) Atomic structure of the grain boundary between grain 9 and 11. (a) View along the $[-1-21]_{9} \|[1-12]_{11}$ direction showing the tilt between the two grains. The regions of coherence in which the close packed $[101]_{9}$ continuously changes to the $[-1-10]_{11}$ direction in grain 11 are highlighted in yellow. These regions are separated by regions of atomic misfit. (b) Intersection of the (c)-plane of grain 9 with the (a)-plane of grain 11 showing the twist misorientation. Within the coherent region (yellow) the slip planes bend and assume locally a close to identical orientation.

in grain 9, a larger partial dislocation half loop $D \alpha(a)_{11}$ appears in grain 11 [see. Figs. 3(c)-3(e)]. This loop expands to a radius of about 9 lattice constants $\left(a_{0}\right)$ into grain 11; however, its ends stay attached to the misfit regions adjacent to the coherent grain boundary region. Subsequently, the loop shrinks and disappears 13 ps after its first appearance. During this entire process the dislocation $A D(c)$ swept the entire grain 9. No further partial dislocation loops were observed in grain 11.

It is interesting to note that no trailing partial dislocation was ever emitted in grain 11. Increasing the applied tensile stress from 1.6 to $1.8 \mathrm{GPa}$ after appearance of $D \alpha(a)_{11}$ leads to a significant increase of loop size, combined with the deposition of dislocation segments on the grain 11 side of the grain boundary. However, the ends of the partial dislocation stayed fixed at the misfit regions. No nucleation of trailing partial dislocation could be observed at the grain boundary. Instead, anomalous partial dislocation nucleation ${ }^{17}$ of $\alpha B(a)_{11}$ took place in the stacking fault. This Burgers vector is favored by the resolved shear stress [for $D B(a)_{11}$ the Schmid factor is $m=0.48$ compared to 0.36 for $D C(a)_{11}$ ].

A closer analysis of the atomistic details of the slip transmission [Figs. 3(b)-3(e)] shows that during the passage of $A D(c)$ in grain 9, the regions of misfit undergo correlated atomic shuffling, resulting in a movement of the coherent regions. The nucleation site of the partial dislocation $D \alpha(a)$ is at the left boarder of the coherent region. The partial dislocation emerged during the passage of the coherent region by the leading partial dislocation $\gamma D(c)$ in grain 9 [see sketch in Fig. 3(f)]. In order to transfer the slip caused by $A D(c)$ in grain 9 to grain 11, either $D B(a)_{11}$ or $D C(a)_{11}$ have to accommodate the slip in grain 11. In both cases the leading partial dislocation has to be $D \alpha(a)_{11}$ in order to create a hcp stacking fault. On the other hand, the nucleation of a trailing partial dislocation [either $\alpha C(a)_{11}$ or $\alpha B(a)_{11}$ ] would create a displacement that destroys the energetically favorable coherent region. This is most probably the reason why the emission of a trailing partial dislocation in grain 11 is not observed in the limited time scale accessible to the MD simulation.

The exact atomic process, i.e., whether the creation of a ledge at the GB by the incoming dislocation leads to the nucleation of the dislocation or whether the incoming Burgers vector is partially transmitted to the neighboring grain while leaving a residual Burgers vector at the grain boundary, could not be unambiguously determined from the atomistic simulations. The dislocation configuration and therefore the resulting atomic displacements would be identical for both mechanisms.

However, several mesoscopic aspects of this slip transmission event are relevant to the understanding and modeling of slip transfer between grains. First, it shows that contrary to the classical description of slip transmission, ${ }^{1}$ direct transmission is possible even though the glide planes in both grains do not intersect along a line at the grain boundary. In the present case, this is a direct consequence of the existence of coherent regions in the grain boundary where the glide planes are locally bent and thus show locally a continuous change of the lattice from one grain to the other. Shen et $a l .{ }^{3,18}$ derived criteria for comparatively easy transmission of slip for general high grain boundaries. These criteria were further developed by Lee et al. ${ }^{19-21}$ who stated that for comparatively easy transmission of slip,

(1) the angle between the lines of intersection of the slip planes in both grains should be as small as possible,

(2) the residual Burgers vector which has to be left in the grain boundary should be small, and

(3) the resolved shear stress on the outgoing slip system should be high.

The observed slip transmission agrees well with these criteria, which were derived from transmission electron mi-
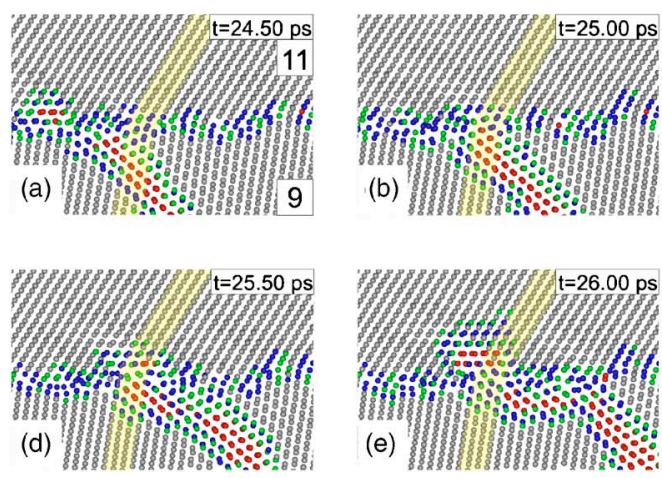
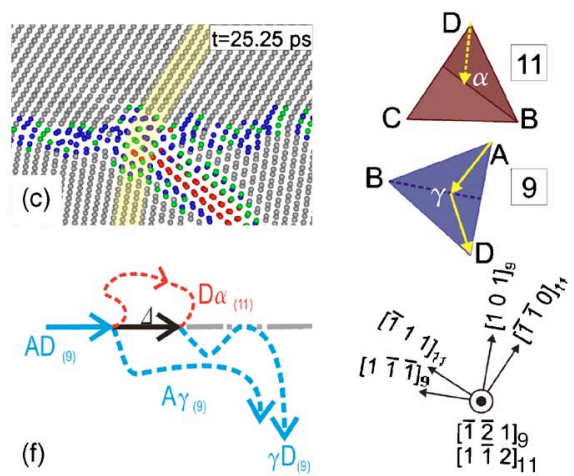

FIG. 3. (Color online) [(a)-(e)] Snapshots of two slip transfer events at coherent regions during the passage of $A D(c)$ in grain 9. The dislocation configuration of (e) is shown schematically in (f). The orientation of the slip systems of the two grains together with the identified Burgers vectors is shown on the Thompson tetrahedra. 
croscopy observations, and thus do not take into account the possible existence of coherent atomic regions within the grain boundary. However, in addition, the atomistic simulation reveals that the regions of misfit act as strong pinning centers for the newly created dislocation. Furthermore, the simulations lead us to the assumption that the choice of the Burgers vector of the outgoing dislocation might not be influenced only by criteria 2 and 3, but also might be determined by its influence on the local atomic structure of the grain boundary. From the above discussion it becomes clear that for the understanding and the modeling of slip transmission through general large angle grain boundaries, the fully three dimensional dislocation/grain boundary geometry, including the local grain boundary structure, i.e., the size and distribution of possible coherent regions, has to be taken into account. This is, however, currently not the case in most multiscale modeling approaches of dislocation/grain boundary interactions, which either deal with two dimensional models ${ }^{7}$ or neglect the internal structure of grain boundaries. ${ }^{17,22,23}$

In conclusion, we have reported on the observation of dislocation transmission through a general high-angle grain boundary in a MD simulation of a three dimensional nanocrystalline aluminum sample under tension. The results point to the importance of the internal grain boundary structure for the transmission of slip, which can only be captured in three dimensional models of dislocation/grain boundary interaction.

The authors acknowledge the financial support of the European Commission (FP6-NANOMESO, No. 016710).

${ }^{1}$ A. P. Sutton and R. W. Balluffi, Interfaces in Crystalline Materials
(Oxford University Press, Oxford, 2006), Section 12.6, p. 737.

${ }^{2}$ K. S. Kumar, H. Van Swygenhoven, and S. Suresh, Acta Mater. 51, 5743 (2003).

${ }^{3}$ Z. Shen, R. H. Wagoner, and W. A. T. Clark, Acta Metall. 36, 3231 (1988).

${ }^{4}$ H. L. Heinisch, R. G. Hoagland, R. J. Kurtz, and J. P. Hirth, Scr. Mater. 39, 451 (1998).

${ }^{5}$ Z. H. Jin, P. Gumbsch, E. Ma, K. Albe, K. Lu, H. Hahn, and H. Gleiter, Scr. Mater. 54, 1163 (2006).

${ }^{6}$ K. J. Kim, J. H. Yoon, M. H. Cho, and H. Jang, Mater. Lett. 60, 3367 (2006).

${ }^{7}$ M. P. Dewald and W. A. Curtin, Modell. Simul. Mater. Sci. Eng. 15, 193 (2007).

${ }^{8}$ H. Jang and D. Farkas, Mater. Lett. 61, 868 (2007).

${ }^{9}$ T. Zhu, J. Li, A. Samanta, H. G. Kim, and S. Suresh, Proc. Natl. Acad. Sci. U.S.A. 104, 3031 (2007).

${ }^{10} \mathrm{H}$. Van Swygenhoven, Science 296, 66 (2002).

${ }^{11}$ J. Schiotz and K. W. Jacobsen, Science 301, 1357 (2003).

${ }^{12}$ P. M. Derlet and H. Van Swygenhoven, Phys. Rev. B 67, 8 (2003).

${ }^{13}$ Y. Mishin, D. Frakas, M. J. Mehl, and D. A. Papaconstantopoulos, Mater. Res. Soc. Symp. Proc. 538, 535 (1999).

${ }^{14}$ J. D. Honeycutt and H. C. Andersen, J. Phys. Chem. 91, 4950 (1987).

${ }^{15}$ J. A. Zimmerman, C. L. Kelchner, P. A. Klein, J. C. Hamilton, and S. M. Foiles, Phys. Rev. Lett. 87, 165507 (2001).

${ }^{16}$ J. P. Hirth and J. Lothe, Theory of Dislocations, 2nd ed. (Wiley, New York, 1982), p. 319.

${ }^{17}$ M. de Koning, W. Cai, and V. V. Bulatov, Phys. Rev. Lett. 91, 025503 (2003).

${ }^{18}$ Z. Shen, R. H. Wagoner, and W. A. T. Clark, Scr. Metall. 20, 921 (1986).

${ }^{19}$ T. C. Lee, I. M. Robertson, and H. K. Birnbaum, Scr. Metall. 23, 799 (1989).

${ }^{20}$ T. C. Lee, I. M. Robertson, and H. K. Birnbaum, Metall. Trans. A 21, 2437 (1990).

${ }^{21}$ T. C. Lee, I. M. Robertson, and H. K. Birnbaum, Philos. Mag. A 62, 131 (1990).

${ }^{22}$ M. de Koning, R. Miller, V. V. Bulatov, and F. Abraham, Philos. Mag. A 82, 2511 (2002).

${ }^{23}$ M. de Koning, R. J. Kurtz, V. V. Bulatov, C. H. Henager, R. G. Hoagland, W. Cai, and M. Nomura, J. Nucl. Mater. 323, 281 (2003). 